\title{
Análisis climático para la agricultura de temporal en Michoacán, México
}

\section{Agro Climatic analysis for rainfed agriculture in Michoacan, Mexico}

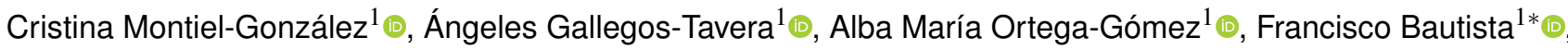 \\ Fernando Gopar-Merino ${ }^{1,2}$ (D), Alejandro Velázquez ${ }^{1}$ (D) \\ ${ }^{1}$ Centro de Investigaciones en Geografía Ambiental, Universidad Nacional Autónoma de México. Antigua Carretera a Pátzcuaro, Col. Ex \\ Hacienda de San José de la Huerta, CP. 58190, Morelia, Michoacán, México. \\ ${ }^{2}$ Centro de Investigación en Ciencias Biológicas Aplicadas, Universidad Autónoma del Estado de México. Carretera Toluca-Ixtlahuaca \\ Km 14.5, CP. 50200. San Cayetano Morelos, Toluca de Lerdo, México. \\ *Autor de correspondencia: leptosol@ciga.unam.mx
}

Artículo científico recibido: 28 de agosto de 2018 aceptado: 18 de enero de 2019

RESUMEN. El estado de Michoacán es uno de los mayores productores de cultivos de temporal con repercusión importante en la economía nacional. A pesar de la importancia agrícola del estado, aun no se han realizado estudios agroclimáticos explícitos que identifiquen el potencial de la Longitud del Periodo de Crecimiento (LPC). El objetivo del estudio fue realizar un análisis agroclimático del estado de Michoacán, considerando la LPC por zonas bioclimáticas. Se calculó la LPC de 112 estaciones meteorológicas situadas dentro del estado, con los datos climáticos se calculó el índice de humedad $(\mathrm{IH})$ utilizando la evapotranspiración por los métodos de Thornthwaite y Hargreaves, se consideró los valores de IH mayores a 0.5 y se identificó el número de meses con lluvia continua, los cuales constituyen la LPC; que se utilizaron para espacializarlos por zonas bioclimáticas en un mapa. El análisis agroclimático mediante el uso de la LPC permitió identificar las zonas con 6 , 5,4 y 3 meses de lluvia continua. Con el mapa generado se pueden proponer el establecimiento de cultivos de temporal por zonas con potenciales climáticos que cubran los requerimientos de los cultivos; además, puede ser de utilidad para enfrentar el cambio climático mediante la sustitución de los cultivos en las zonas acordes a los meses de lluvia agrícola continua.

Palabras clave: Cultivo de temporal, duración de periodo de lluvias, índice agroclimático, longitud de periodo de crecimiento.

ABSTRACT. Michoacán is among the states with higher rainfed agriculture production in México, and has substantial impact on the national economy. Despite the agricultural importance of this state, no agroclimatic studies have been perform to identify the Length of the Growing Period (LGP), which is the number of months when the climatic conditions are suitable for the establishment of rainfed agriculture. In the present study, we aimed to perform an agroclimatic analysis for the state of Michoacán to determine the LGP for bioclimatic zones. In order to achieve our objective, we calculated the LGP for 112 meteorological stations located within Michoacán. We calculated the humidity index (HUi) using evapotranspiration by two methods: 1) Thornthwaite ( $\left.E T_{0}-\mathrm{T}\right)$ and 2) Hargreaves $\left(\mathrm{ET}_{0}-\mathrm{H}\right)$. The values with a HUi higher than 0.5 were used to determine the number of months with continuous rainfall, which constitute the LGP; these data were extrapolated to a map of bioclimatic zones. As a result, the agroclimatic analysis to determine the LGP, allowed us to identify the zones with $6,5,4$ and 3 months of continuous rainfall. Based on the resulting map, we can to recommend areas with climatic potentials suitable to the requirements of the crops. Our map could also prove useful in the face of climate change by allowing us to relocate the rainfed crops to the zones with an adequate number of months of continuous rainfall.

Key words: Agroclimatic index, continuous rainfall, rainfed agriculture, length of the growing period.

\section{INTRODUCCIÓN}

De acuerdo con el Fondo de Población de las Naciones Unidas (UNFPA 2018), en el 2017 la población mundial alcanzó los 7600 millones de personas. De continuar con la tasa de crecimiento poblacional actual, se proyecta que para el 2050 la población podría llegar a los 9770 millones (ONU 
2017). Este aumento considerable de la población favorecerá un incremento en la demanda de recursos provenientes de actividades agrícolas, lo cual aumentará la posibilidad de una crisis alimentaria, en especial en países subdesarrollados (Alexandratos y Bruinsma 2012).

Adicionalmente, se sabe que los sistemas de producción agrícola son altamente vulnerables ante los efectos del cambio climático (Kang y Banga 2013). Debido a los cambios en los regímenes de precipitación y temperatura que se han traducido en sequías, lluvias torrenciales, granizadas, ondas de calor y heladas que dañan la producción de los cultivos (IPCC 2012).

México se encuentra en el décimo primer lugar de producción agrícola del mundo, mientras que el estado de Michoacán, con una producción de 157 cultivos, es uno de los mayores productores agrícolas del país (SIAP 2017b), aportando el 2.2 y $4.5 \%$ del producto interno bruto (PIB) nacional y estatal, respectivamente (SFA 2018). De los cultivos producidos en el estado, el $40.5 \%$ se cultiva bajo riego y el $59.5 \%$ bajo temporal (SIAP 2017b).

La variación de la precipitación podría tener efectos directos en la producción de los cultivos de temporal. De acuerdo con el atlas de cambio climático para el estado de Michoacán (FernándezEguiarte et al. 2010), se propusieron escenarios de cambio climático desarrollados con el modelo MPI ECHAM 5 A2 para meses lluviosos (junio y septiembre); en el que se indica que para el 2050 la precipitación promedio podría disminuir hasta en $20 \mathrm{~mm}$ en municipios al suroeste del estado de Michoacán, mientras que, para el resto de los municipios se proyecta un aumento de hasta $20 \mathrm{~mm}$. Además, del incremento de la temperatura promedio de entre 1.8 y $2.3^{\circ} \mathrm{C}$ y de 1.2 a $1.4^{\circ} \mathrm{C}$ para los meses de junio y septiembre, respectivamente. Actualmente se tiene la zonificación con taxonomía de climas (INEGI 2018), así como la zonificación bioclimática a escala regional (Gopar-Merino et al. 2015, Hernández et al. 2018). Pero no se tienen estudios agroclimáticos en los que se identifique el potencial de la Longitud del Periodo de Crecimiento (LPC) (FAO 1996). Lo anterior tiene importancia práctica, principalmente ante los posibles cambios en el régimen térmico y de precipitación pluvial debido al cambio climático, así como para el establecimiento de nuevos cultivos o por la apertura de nuevas tierras para la agricultura (Vrieling et al. 2013).

Se han propuesto valores agroclimáticos umbrales a partir de los cuales se infiere que las condiciones climáticas son óptimas para el desarrollo de los cultivos de temporal (Bautista et al. 2016). Dentro de estos valores se encuentran: 1) temperatura mínima mayor a $5^{\circ} \mathrm{C}$, para asegurar que se superan temperaturas de heladas y ondas de frio que puedan afectar el desarrollo de los cultivos (Snyder y MeloAbreu 2010); y 2) el índice de LPC (FAO 1996, Bautista et al. 2009, Delgado et al. 2017). Por lo anterior, el objetivo del presente trabajo fue desarrollar una regionalización basada en datos climohidrográficos y la Longitud del Periodo de Crecimiento para identificar las áreas con potencial de albergar cultivos de temporal.

\section{MATERIALES Y MÉTODOS}

\section{Área de estudio}

El estudio se llevó al cabo en el Estado de Michoacán en el que confluyen climas templados, tropicales húmedos y tropicales secos. El estado se localiza en el occidente de México, entre las coordenadas $20^{\circ} 24^{\prime}$ a $17^{\circ} 55^{\prime} \mathrm{LN}$, y $100^{\circ} 04^{\prime}$ a $103^{\circ} 44^{\prime}$ LO; tiene 113 municipios, con una extensión de 58 $643.3 \mathrm{~km}^{2}$ que representan el $3.0 \%$ del territorio nacional (SIAP 2017b). Colinda al norte con los estados de Jalisco y Guanajuato, al noreste con Querétaro, al este con Estado de México, al sureste con Colima y al Sur con Guerrero. Se encuentra ubicado dentro de las provincias geológicas denominadas Sierra Madre del Sur y Sistema Volcánico Transversal; alberga cinco unidades naturales: Costera, Sierra Madre del Sur, Depresión del Balsas-Tepalcatepec, Sistema Volcánico Transversal y Altiplanicie Mexicana (Antaramián y Correa 2003).

De acuerdo con la clasificación climática modificada por García (1981), predominan cuatro tipos de clima: 1) en el suroeste Aw, tropical lluvioso con lluvias predominantes en verano, 2) en la depre- 
sión del río Tepalcatepec BS, seco estepario, 3) En el norte $\mathrm{Cw}$, templado con lluvias en verano y 4) En las partes más altas del sistema Volcánico Transversal $\mathrm{Cf}$, templado con lluvias todo el año (Antaramián 2005). Los grupos de suelo predominantes son: Leptosols, Regosols, Luvisols, Acrisols, Andosols, Vertisols y Faozems y en menor proporción Cambisols, Fluvisols, Planosols, Gleysols, Solonchacks, Kastañozems e Histosols (Cabrera et al. 2005). De acuerdo con Carranza (2005) las comunidades vegetales presentes en el estado son: 1) bosque de coníferas, dentro de los que se encuentran bosque de pino, bosque de oyamel y bosque de cedro blanco; 2 ) Bosque de encino, 3) Bosque mesófilo de montaña, 4) Bosque tropical caducifolio, 5) Matorral subtropical, 6) Bosque espinoso, 7) Bosque tropical subcaducifolio, 8) Vegetación acuática y subacuática entre las que se encuentra tular y carrizal, bosque de galería y manglar, y 9) Otros tipos de vegetación entre los que destacan palmar, pastizal y vegetación de dunas costeras.

\section{Bases de datos}

Se elaboró una base de datos de precipitación pluvial mensual $(\mathrm{P})$, temperaturas máximas (Tmax), temperaturas medias (Tmed) y temperaturas mínimas (Tmin) con el software Clic-MD 2.0 (Bautista et al. 2016). Los datos se obtuvieron de 112 estaciones meteorológicas (Figura 1) ubicadas en el estado de Michoacán, México, que pertecen al Servicio Meteorológico Nacional (SMN 2018). Para la selección de las bases de datos empleadas en los análisis, se uso el criterio de que tuvieran datos continuos por más de 10 años. La información de las variables climáticas se empleó para: 1) calcular la evapotranspiración potencial $\left(\mathrm{ET}_{0}\right)$ con los métodos de Thornthwaite (Thornthwaite 1948) y Hargreaves (Hargreaves et al. 1985, Hargreaves y Samani 1985), y 2) para calcular la longitud del periodo de crecimiento (LPC) (Bautista et al. 2009, Delgado et al. 2017). El cálculo de la LPC se realizó para cada estación meteorológica con el software Clic-MD 2.0, con la misma fórmula empleada para el cálculo del índice de humedad $\mathrm{IH}$, la cual fue:

$$
L C P=I H=\frac{P}{E T_{0}}
$$

Dónde: $\mathrm{LPC}=$ Longitud del periodo de crecimiento, $\mathrm{P}=$ Precipitación pluvial, y $\mathrm{ET}_{0}=$ Evapotranspiración potencial estimada con los métodos tanto de Thornthwaite $\left(\mathrm{ET}_{0}-\mathrm{T}\right)$, como de Hargreaves $\left(\mathrm{ET}_{0}-\mathrm{H}\right)$. Se identificó el mes de inicio y el mes de finales de lluvias y se contabilizó el número de meses continuos con lluvia, tomado a partir del valor de Hui mayor a 0.5 con $\mathrm{ET}_{0}-\mathrm{T}$ y $\mathrm{ET}_{0}-\mathrm{H}$, para reportar la LPC.

\section{Análisis espacial}

Para el análisis espacial se generó una base de datos georreferenciada de cada estación meteorológica utilizada, la selección, filtro de la calidad y cantidad de estaciones meteorológicas se realizó con las bases de datos del INIFAP (2018) y el Atlas Climático Digital de México (FernándezEguiarte et al. 2018). Con base en Gopar-Merino et al. (2015) y con el Atlas Climático Digital de México, se generaron las capas de los promedios mensuales y anuales de precipitación de 1902 a 2011. Las capas raster se analizaron en el SIG ArcGIS 9.3, con el que se realizó el cálculo de los diversos índices bioclimáticos que fueron la base para la construcción de las diferentes categorías climo-hidrográficas denominadas ombrotipos y que cubren toda el área de estudio. En total se definieron cinco ombrotipos (semiárido, seco, subhúmedo, húmedo e hiperhúmedo), validados por la información de las estaciones meteorológicas y por información de 196 sitios de trabajo de campo. En la Tabla 1 se muestran la extensión en $\mathrm{km}^{2}$ de cada uno de los 14 isobioclimas para el estado de Michoacán descritos taxonómicamente por bioclima, termotipo y ombrotipo (GoparMerino et al. 2015).

La representación cartográfica combinando la longitud del periodo de crecimiento (LPC) y la zonificación ombrotérmica validada, generó la zonificación final. La determinación de los meses de la longitud del periodo de crecimiento (LPC) por ombrotipo se realizó con un script en Python que selecciona el valor identificado como la moda de la LPC (Delgado et al. 2017), y cuantifica el número de estaciones por ombrotipo. Los mapas se editaron con el Sistema de Información Geográfica Arc GIS 10.5 (Esri 2018). 


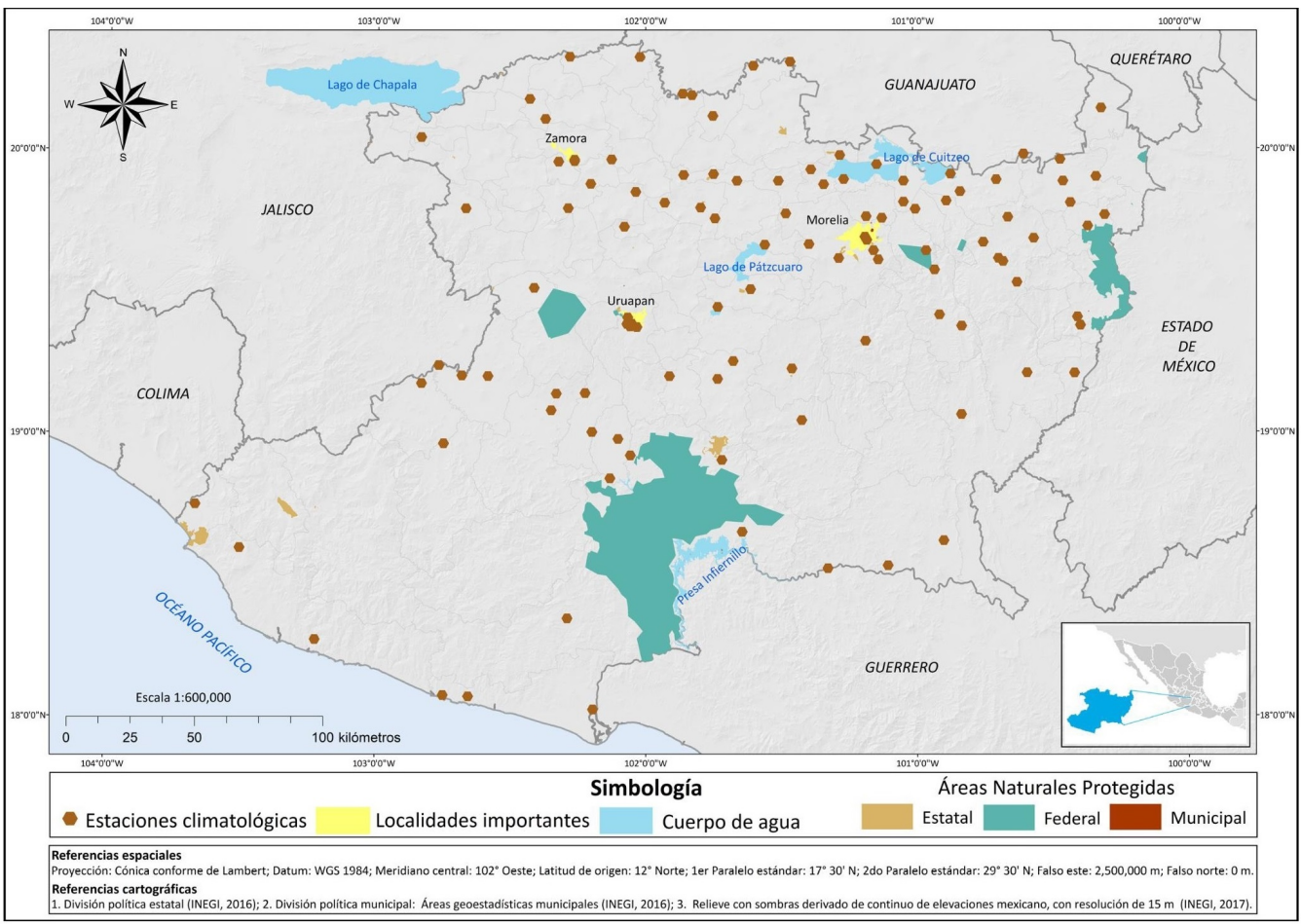

Figura 1. Ubicación de las estaciones meteorológicas y áreas naturales protegidas (ANP) dentro del estado de Michoacán. Los puntos naranjas indican la ubicación de las 112 estaciones climáticas de las que se extrajeron los datos climáticos. Los polígonos indican la delimitación de las áreas naturales protegidas en el estado de Michoacán, se muestran localidades importantes y cuerpos de agua.

Tabla 1. Extensión en $\mathrm{km}^{2}$ de los 14 Isobioclimas del Estado de Michoacán con descripción taxonómica por bioclimas, termotipo y ombrotipo.

\begin{tabular}{llccccc}
\hline \multirow{2}{*}{ Bioclima } & Termotipo & \multicolumn{5}{c}{ Ombrotipo } \\
\cline { 3 - 7 } & & Híper-húmedo & Húmedo & Subhúmedo & Seco & Semiárido \\
\hline Pluvial & Supratropical & 1.07 & 1.36 & - & - & - \\
Pluviestacional & Infratropical & - & 29.05 & 1057.08 & - & - \\
& Termotropical & - & 539.38 & 11479.15 & - & - \\
& Mesotropical & - & 6417.63 & 12393.45 & - & - \\
\multirow{2}{*}{ Xérico } & Supratropical & 24.81 & 819.36 & - & - & - \\
& Infratropical & - & - & - & 14177.89 & 1562.54 \\
& Termotropical & - & - & - & 7858.55 & - \\
& Mesotropical & - & - & - & 1968.26 & - \\
\hline
\end{tabular}

\section{RESULTADOS}

\section{Mapa agroclimático de la LPC}

La distribución espacial de la LPC en el mapa muestra un gradiente de menor (tres meses) a mayor número de meses (seis meses) en dirección de sur a centro y de norte a centro del estado de Michoacán (Tabla 2).
Ombrotipos pluviestacionales 5 a 6 meses de LPC

La mayor LPC en los ombrotipos pluviestacionales fue de seis meses que se observó en el $11.5 \%$ de la superficie del estado (Figura 2 ). Un ejemplo es la estación Los azufres en la que durante seis meses se supera el valor de 0.5 del índice humedad. Siendo el mes de julio muy húmedo, y los meses de agosto, septiembre y octubre hiperhúmedos (Figura 3). 
Montiel-González et al.

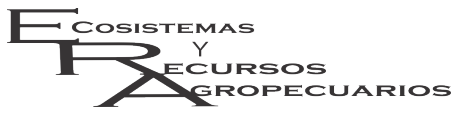

Agro Climatic analysis for rainfed agriculture

Ecosist. Recur. Agropec.

6(17):307-316,2019

Tabla 2. Lista de 11 ombrotipos identificados en el estado de Michoacán. Se indica la LPC en meses, inicio y fin de la LPC, número de estaciones con las que se determinó y la superficie que abarca cada isobioclima en hectáreas.

\begin{tabular}{lcclrr}
\hline Isobioclima & $\begin{array}{c}\text { LPC } \\
(\mathrm{meses})\end{array}$ & $\begin{array}{c}\text { Mes de } \\
\text { inicio* }\end{array}$ & $\begin{array}{l}\text { Mes de } \\
\text { fin* }^{*}\end{array}$ & $\begin{array}{c}\text { No. de } \\
\text { Estaciones }\end{array}$ & $\begin{array}{r}\text { Área } \\
(\mathrm{km} 2)\end{array}$ \\
\hline Pluviestacional supratropical húmedo & 6 & Junio & Noviembre & 1 & 569.75 \\
Pluviestacional mesotropical húmedo & $6-5$ & Mayo & Octubre & 5 & 6174.32 \\
Pluviestacional termotropical húmedo & 5 & Junio & Octubre & 6 & 539.34 \\
Pluviestacional infratropical húmedo & 5 & Junio & Octubre & 1 & 28.98 \\
Pluviestacional mesotropical subhúmedo & 5 & Junio & Octubre & 42 & 12251.94 \\
Pluviestacional termotropical subhúmedo & 5 & Junio & Octubre & 5 & 11392.11 \\
Pluviestacional infratropical subhúmedo & 5 & Junio & Octubre & 1 & 1057.08 \\
Xérico mesotropical seco & 4 & Junio & Septiembre & 9 & 1961.12 \\
Xérico termotropical seco & 4 & Junio & Septiembre & 16 & 7356.46 \\
Xérico infratropical seco & 4 & Junio & Septiembre & 18 & 12698.78 \\
Xérico infratropical semiárido & 3 & Julio & Septiembre & 8 & 874.5 \\
\hline
\end{tabular}

En el cálculo de la LPC: para los ombrotipos Pluviestacional se utilozó $\mathrm{ET}_{0}-\mathrm{H}$ mientras que para los ombrotipos Xérico se utilizó la $\mathrm{ET}_{0}-\mathrm{T}$. El * indica mes de inicio y mes de fin de temporada de lluvia continua de los meses mas frecuentes a partir de la moda estadística.

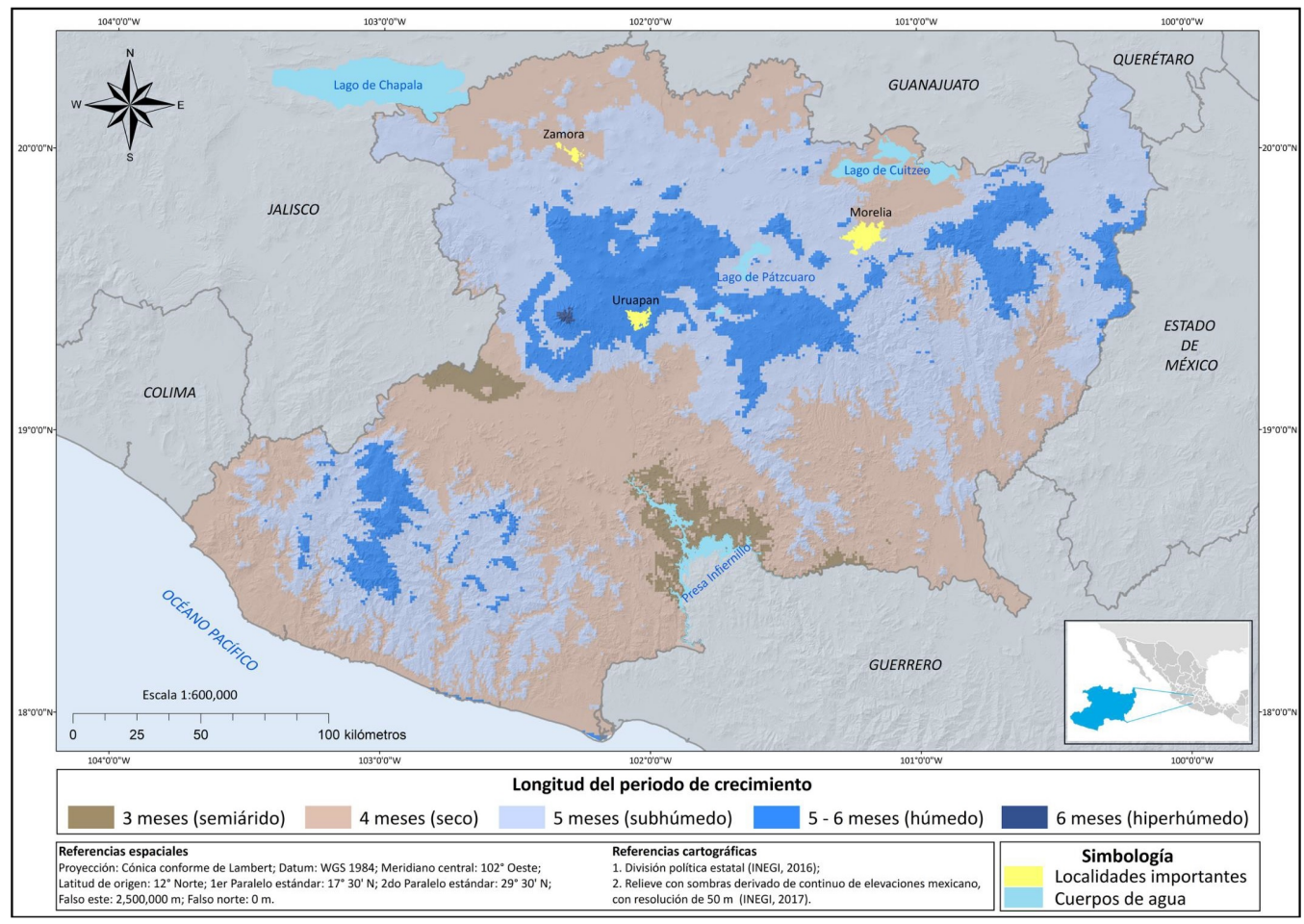

Figura 2. Mapa de la longitud del periodo de crecimiento (LPC) para el estado de Michoacán.

Los ombrotipos pluviestacionales con cinco meses de LPC ocupan una superficie del $43.1 \%$ del estado. Por ejemplo, en la estación meteorológica Maravatío se tienen tres meses húmedos y uno muy húmedo. Los ombrotipos Pluviestacional supratropical húmedo, Pluviestacional infratropical húmedo y Pluviestacional infratropical subhúmedo son los que contienen solo una estación meteorológica, por lo que son los de mayor incertidumbre, pero también son los de ocupan menor superficie de los ombrotipos pluviestacionales (Tabla 2). 

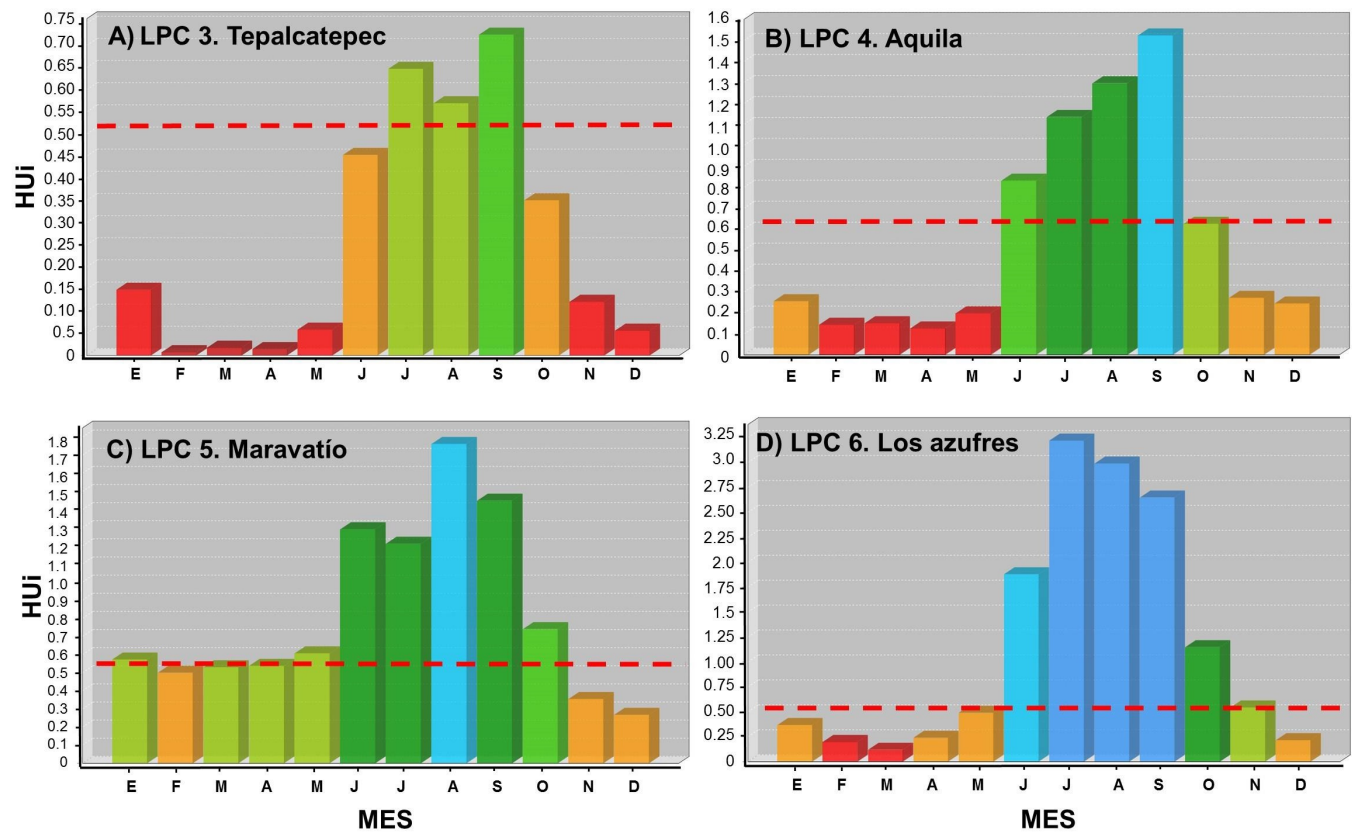

\#iperárida Árida Semiárida @ Subhúmeda-seca

Húmeda Muy húmeda Hiperhúmeda

Figura 3. Índice de Humedad (HUi) por mes para cuatro municipios de importancia agrícola a nivel estatal. Los gráficos ejemplifican la longitud del periodo de lluvias (LPC) cuando el Hui es mayor a 0.5 , el cual se indica en líneas rojas. Para el LPC 5 y 6 se utilizó $\mathrm{ET}_{0}-\mathrm{H}$ mientras que para LPC 3 y 4 se utilizó la $\mathrm{ET}_{0}-\mathrm{T}$.

Cuando se considera el inicio y termino de la LPC, el isobioclima con mayor incertidumbre es el Pluviestacional mesotropical húmedo, ya que en el $60 \%$ de las estaciones meteorológicas la LPC comienza en mayo, mientras que en el restante $40 \%$ comienza en junio (Tabla 3 ). En todos los demás casos la LPC comienza en junio en más del $80 \%$ de las estaciones meteorológicas estudiadas. En el isobioclima pluviestacional mesotropical subhúmedo el término de la LPC se presenta en septiembre en el $38 \%$, mientras que en el $62 \%$ de los casos en el mes de octubre. Este isobioclima es el de menor certidumbre de los pluviestacionales.

\section{Los ombrotipos xéricos 3 a 4 meses de LPC}

El valor de la moda de la LPC en los ombrotipos xéricos osciló entre 4 y 3 meses. La superficie con LPC de 4 meses comprende el $37.5 \%$ del territorio estatal (Figura 2). El menor valor de la LPC correspondió a tres meses. La LPC 3 se registró en el clima más seco, el isobioclima xérico infratropical semiárido, que ocupa una superficie del $1.5 \%$ del territorio estatal (Figura 2). La Figura 3 muestras la estación meteorológica Tepalcatepec en la que en los meses de julio a septiembre se superan el valor de 0.5 del índice de humedad. Para los ombrotipos xéricos, en el infratropical seco la LPC termina en septiembre en el $62 \%$ de los casos y excepcionalmente llega hasta el mes de octubre en el $30 \%$ de los casos (Tabla 3).

\section{DISCUSIÓN}

De las 256 estaciones registradas en el sistema CLICOM (2018), sólo 112 cumplieron con los criterios de validación del presente estudio. En la Figura 1 se muestra que en el estado de Michoacán las estaciones se encuentran concentradas en la parte norte del estado, en menor medida en el centro y pocas en el suroeste. Los municipios el Aguililla y Apatzingán, son poco monitoreados, aun cuando son productores de diversos cultivos de temporal de im- 
Tabla 3. Distribución anual del porcentaje de estaciones en las que se registró el mes de inicio y final del periodo de lluvias o longitud de periodo de crecimiento (LPC) calculado con diferentes métodos.

\begin{tabular}{|c|c|c|c|c|c|c|c|}
\hline \multirow{2}{*}{ Isobioclima } & \multirow{2}{*}{$\begin{array}{c}\text { LCP } \\
\text { (meses) }\end{array}$} & \multicolumn{3}{|c|}{ Mes de inicio (\%)) } & \multicolumn{3}{|c|}{ Mes de Final (\%) } \\
\hline & & Mayo & Junio & Julio & Septiembre & Octubre & Noviembre \\
\hline Pluviestacional supratropical húmedo & 6 & - & 100 & - & - & - & 100 \\
\hline Pluviestacional mesotropical húmedo & $6-5$ & 60 & 40 & - & - & 80 & 20 \\
\hline Pluviestacional termotropical húmedo & 5 & 10 & 90 & - & - & 90 & 10 \\
\hline Pluviestacional infratropical húmedo & 5 & - & 100 & - & - & 100 & - \\
\hline Pluviestacional mesotropical subhúmedo & 5 & 5 & 95 & - & 38 & 62 & - \\
\hline Pluviestacional termotropical subhúmedo & 5 & 20 & 80 & - & 20 & 80 & - \\
\hline Pluviestacional infratropical subhúmedo & 5 & - & 100 & - & - & 100 & - \\
\hline Xérico mesotropical seco & 4 & - & 100 & - & 89 & 11 & - \\
\hline Xérico termotropical seco & 4 & - & 100 & - & 82 & 18 & - \\
\hline Xérico infratropical seco & 4 & & 95 & 5 & 62 & 38 & - \\
\hline Xérico infratropical semiárido & 3 & - & 25 & 75 & 100 & - & - \\
\hline
\end{tabular}

portancia nacional (SIAP 2017a, SIAP 2018). Lo que refuerza el valor de la elaboración de mapas agroclimáticos regionales.

\section{Mapa agroclimático de la LPC}

Los ombrotipos se organizaron para validar que la LPC concordara de forma satisfactoria con el tipo de Isobioclima. Con los análisis se determinó que el método más adecuado para estimar la $\mathrm{ET}_{0}$ en climas semiáridos es el de Thornthwaite (Thornthwaite 1948), mientras que el más adecuado para climas húmedos es el de Hargreaves (Hargreaves y Samani 1985), debido a que ambos métodos se ajustan mejor a los valores esperados. Al respecto la FAO (1996) propone que el índice de humedad $(\mathrm{IH})$ debe determinarse con el cociente de $\mathrm{P} / \mathrm{ET}_{0}>0.5$, indistintamente de la forma de calcular la evapotranspiración. En el presente estudio se observó que el mejor predictor de la LPC por IH, es la evapotranspiración $\left(\mathrm{ET}_{0}\right)$ calculada con el método de Thornthwaite para los ombrotipos xéricos, mientras que para los ombrotipos pluviestacionales el mejor predictor de la LPC es el cálculo de la $\mathrm{ET}_{0}$ con el método de Hargreaves. Estos resultados son consistentes con lo reportado en estudios que determinan la relación entre el cálculo de la $\mathrm{ET}_{0}$ y la LPC (Bautista et al. 2009, Delgado et al. 2017). El mapa generado puede servir de base para la planeación agrícola, ya que la precisión en el cálculo de la LPC es consistente con los ombrotipos y la precisión es alta en la mayor superficie del estado (Gopar-Merino et al. 2015). La incer- tidumbre se localiza en los polígonos de transición bioclimática en los cuales el número de las estaciones meteorológicas es bajo. También depende de la variabilidad climática observada en algunos ombrotipos, la cual podría estar asociada al cambio climático en esas zonas de transición bioclimática (Gallego-Sala et al. 2010). Una forma de mejorar el cálculo de la LPC es mediante la calibración de la $\mathrm{ET}_{0}$ es ajustando las ecuaciones de Thornthwaite (Thornthwaite 1948) y Hargreaves (Hargreaves et al. 1985) mediante la comparación con la ecuación de Penman-Monteith que propone la FAO (Allen et al. 1998), y usando el método propuesto por Bautista et al (2009). Con la $\mathrm{ET}_{0}$ recalibrada también se podría calcular el uso consuntivo de agua por cada cultivo (Allen et al. 1998).

\section{Ombrotipos pluviestacionales 5 a 6 meses de LPC}

En el estado de Michoacán la producción de los cultivos de temporal de primavera a verano se divide en dos grupos: 1) cultivos anuales en los que la producción depende de forma exclusiva del ingreso de agua por lluvias, y 2) cultivos perennes en los que parte importante de su productividad depende de las lluvias de temporal y el resto del año pueden o no ser mantenidos con riego (SIAP 2017ab, SIAP 2018). AI comparar el mapa de la LPC (Figura 2) con las zonas de producción de cultivos de temporal en el estado de Michoacán para el 2017, se propone la relación de la distribución de cultivos de temporal por zonas con los mismos periodos de duración de lluvia continua o 
LCP.

La calabacita italiana y aguacate Hass se producen en zonas con la LPC más alta o zonas más húmedas del estado (SIAP 2018), en las cuales la LPC va de cinco a seis meses, con inicio de lluvias en junio o mayo y fin de lluvias de octubre o noviembre. La manzana criolla y la pera se producen en zonas con LPC de cinco meses, con inicio de lluvia en mayo o junio, y fin de lluvias en octubre o septiembre. Mientras que la avena forrajera, cebolla blanca, chile verde (serrano y de árbol), frijol (peruano y flor de junio), maíz grano blanco, papa blanca, pepino chino, sorgo grano, tomate rojo, tomate verde y durazno diamante se producen en las zonas con LPC de cuatro a cinco meses (SIAP 2017ab, SIAP 2018). En estas zonas la lluvia suele iniciar en mayo o junio, y finaliza entre septiembre y octubre.

En México solo algunos estados cuentan con un análisis agroclimático para agricultura de temporal en el que se considera la LPC, algunos de los que ya tienen este tipo de estudios se encuenran Yucatán (Delgado et al. 2011, Delgado et al. 2017) y Guanajuato (Granados et al. 2004), aún y cuando se cuenta con datos de evapotraspiración para todo el país (Lobit et al. 2018) y con las herramientas informáticas para hacerlo de forma ágil y precisa (Bautista et al. 2016).

\section{Los ombrotipos xéricos 3 a 4 meses de LPC}

Las zonas de 3 a 4 meses de lluvia continua son de alta variabilidad en la precipitación pluvial y por lo tanto de alto riesgo para la agricultura de temporal. Pero hay cultivos de alta demanda que se pueden cultivar en estas zonas, como por ejemplo la Jamaica. Mientras que el ajonjolí, brócoli, lechuga, sandia y sorgo forrajero en verde se producen en zonas con LPC de cuatro meses. En estas zonas el inicio de lluvias es en junio, en raras ocasiones en julio y el fin de lluvias entre. septiembre y octubre.

En el presente estudio se observó que hay municipios en el estado de Michoacán, en los que la producción de cultivos de temporal se ve concentrada y localizada. Estos municipios son Aguililla, Apatzingán, Coahuayana, Huetamo, Jiquilpan, Lázaro Cárdenas, La Huacana, La piedad, Morelia, Pátzcuaro, Uruapan Zamora y Zitácuaro (SIAP 2018). Al respecto la propuesta del mapa indica que existe un amplio territorio en el Estado que tienen las condiciones climáticas potenciales para la producción de cultivos de temporal. Para realizar una planificación agrícola eficiente es necesario generar información cartográfica geo-edafológica confiable además de la agroclimática, ya que con ambos mapas de geomorfología-suelos y LPC es posible identificar las zonas agroecológicas que son de gran utilidad para aprovechar de manera sustentable el territorio (FAO 1996).

\section{CONCLUSIONES}

El análisis agroclimático con la Longitud del periodo de crecimiento y la cartográfica del mapa de los ombrotipos identificó las zonas de 6, 5, 4 y 3 meses de lluvia continua, que puede ser útil para la agricultura de temporal. El mapa de la longitud del periodo de crecimiento sirve de base para mejorar la agricultura de riego en aquellos cultivos en los que se conozca el uso consuntivo de agua.

\section{AGRADECIMIENTOS}

Al fondo sectorial CONACYT-SEMARNAT por el apoyo económico al proyecto "Prospección territorial ante escenarios de cambio climático en cuencas de alta vulnerabilidad: bases para el manejo de información y la integración intersectorial" (Clave 0263006) y al fondo CONACYT-CIENCIA BÁSICA (283135). Por las becas posdoctorales de C.M.G., A.M.O.G. y L.G.M. y por el apoyo a la beca doctoral de A.G.

\section{LITERATURA CITADA}

Alexandratos N, Bruinsma J (2012) World agriculture towards 2030/2050: the 2012 revision. Agricultural Development Economics Division. Food and Agriculture Organization of the United Nations. ESA Working Paper No. 12-03. Rome, Italy. 154p. 
Allen RG, Pereira LS, Raes D, Smith M (1998) Crop evapotranspiration-Guidelines for computing crop water requirements-FAO Irrigation and drainage paper 56. FAO, Rome 15p.

Antaramián HE, Correa GP (2003) Atlas Geográfico de Michoacán. Editorial EDDISA. Secretaría de Educación Pública en Michoacán, Universidad Michoacán de San Nicolás de Hidalgo. México. 145p

Antaramián HE (2005) Descripción física y biótica de Michoacán. In: Velasco GL (ed). La biodiversidad en Michoacán estudio de estado. Comisión Nacional para el Conocimiento y uso de la Biodiversidad. Gobierno del estado de Michoacán. México 268p.

Bautista F, Bautista D, Delgado-Carranza C (2009) Calibration of the equations of Hargreaves and Thornthwaite to estimate the potential evapotranspiration in semi-arid and subhumid tropical climates for regional applications. Atmosfera 22: 331-348.

Bautista F, Pacheco A, Bautista-Hernández D (2016) Climate change analysis with monthly data (Clic-MD). Editorial Skiu. México 57p

Cabrera GA, González CJC, Ayala GJM (2005) Descripción física y biótica: Los suelos. In: Velasco GL (ed). La biodiversidad en Michoacán estudio de estado.Comisión Nacional para el Conocimiento y uso de la Biodiversidad. Gobierno del estado de Michoacán. México. pp: 29-31.

Carranza GE (2005) Descripción física y biótica: vegetación. Descripción física y biótica: Los suelos. In: Velasco GL (ed). La biodiversidad en Michoacán estudio de estado.Comisión Nacional para el Conocimiento y uso de la Biodiversidad. Gobierno del estado de Michoacán. México. pp: 38-45.

CLICOM (2018) Datos climáticos diarios del CLICOM del SMN a través 268p de su plataforma web del CICESE. http://clicom-mex.cicese.mx/mapa.html. Fecha de consulta: 2 de mayo del 2018.

Delgado C, Bautista F, Ihl T, Palma-López D (2017) Duración del periodo de lluvias y aptitud de tierras para la agricultura de temporal. Ecosistemas y Recurursos Agropecuarios 4: 485-497.

Delgado C, Bautista F, Orellana-Lanza R, Reyes-Hernández H (2011) Classication and agroclimatic zoning using the relationship between precipitation and evapotranspiration in the state of Yucatan, Mexico. Investigaciones Geográcas 75: 51-60.

Esri (2018) ArcGIS Desktop. http://desktop.arcgis.com/es/. Fecha de consulta: 2 de mayo del 2018.

FAO (1996) Agro-Ecological zoning. Guidelines. FAO Soils Bulletin 76. Rome, Italy. 96p

Fernández-Eguiarte A, Romero CR, Zavala-Hidalgo J, Calderón BO, Mendoza PE, Castelán HC, et al. (2010) Atlas de cambio climático del Estado de Michoacán. Michoacán, México: Gobierno del estado de Michoacán, México. 37p.

Fernández-Eguiarte A, Zavala-Hidalgo J, Romero CR (2018) Atlas Climático Digital de México. Versión 2.0. http://uniatmos.atmosfera.unam.mx/ACDM/servmapas2018. Fecha de consulta: 8 de agosto 2018.

Gallego-Sala AV, Clark JM, House JI, Orr HG, Prentice IC, Smith P, et al. (2010) Bioclimatic envelope model of climate change impacts on blanket peatland distribution in Great Britain. Climate Research 45: 151-162.

García E (1981) Modificación al sistema de clasificación climática de Köppen. Instituto de Geografía, Universidad Naional Autónoma de México. México 91p.

Gopar-Merino LF, Velázquez A, de Azcárate JG (2015) Bioclimatic mapping as a new method to assess effects of climatic change. Ecosphere 6: Art13. Doi: 10.1890/ES14-00138.1

Granados R, Reyna T, Soria J, Fernández Y (2004) Aptitud agroclimática en la Mesa Central de Guanajuato, México. Investigaciones Geográficas 54: 24-35. 
Hargreaves GL, Hargreaves GH, Riley JP (1985) Irrigation Water Requirements for Senegal River Basin. Journal of Irrigation and Drainage Engineering 111: 265-275.

Hargreaves HG, Samani AZ (1985) Reference crop evapotranspiration from temperature. Applied Engineering in Agriculture 1: 96-99.

Hernández CME, Ordoñez DMdJ, Giménez AJ (2018) Comparative analysis of two bioclimatic classification systems applied in Mexico | Análisis comparativo de dos sistemas de clasificación bioclimática aplicados en México. Investigaciones Geograficas 95: 1-14.

INEGI (2018) Climatología. Instituto Nacional de Estadística y Geografía. http://www.beta.inegi.org.mx/temas/ mapas/climatologia/. Fecha de consulta: 3 de mayo del 2018.

INIFAP (2018) Red Nacional de Estaciones Agrometeorológicas Automatizadas INIFAP. http://clima.inifap.gob.mx/ Inmysr/Estaciones/MapaEstaciones. Fecha de consulta: 2 de mayo del 2018.

IPCC (2012) Summary for policymakers. In: Field CB, Barros V, Stocker TF, Qin D, Dokken DJ, Ebi KL, et al. (eds.) Managing the risks of extreme events and disasters to advance climate change adaptation. A special report of working groups I and II of the Intergovernmental Panel on Climate Change. Cambridge University Press. Cambridge, UK, and New York, USA. pp: 1-19.

Kang MS, Banga SS (2013) Global Agriculture and Climate Change. Journal of Crop Improvement 27: 667-692.

Lobit P, Gómez-Tagle A, Bautista F, Lhomme J (2018) Retrieving air humidity, global solar radiation and potential evapo-transpiration from daily temperatures: development and validation of new methods for Mexico. Part III: Potential evapo-transpiration. Theoretical and Applied Climatology 133: 787-797.

ONU (2017) World population prospects: The 2017 revision. Volumen II: Demographic Profiles. United Nations. New York, USA. 848p.

SFA (2018) Indicadores Estatales Agroeconómicos. Subcecretaría de Fomento a los Agronegocios de la SAGARPA. http://www.sagarpa.gob.mx/agronegocios/Estudios/Paginas/monitoreoestatal.aspx. Fecha de consulta: 10 de agosto del 2018.

SIAP (2017a) Atlas Agro alimentario 2017. SAGARPA. México. 232p

SIAP (2017b).Infonografía Agroalimentaria 2017. SAGARPA. México. 52p.

SIAP (2018) Avance de Siembras y Cosechas, Resumen por Estado. Servicios de Información Agroalimentaria y Pesquera. http://infosiap.siap.gob.mx:8080/agricola_siap_gobmx/ResumenProducto.do. Fecha de consulta: 3 de mayo del 2018.

SMN (2018) Estaciones meteorológicas de México. Servicio Meteorológico Nacional. http://smn.conagua.gob.mx/ es/climatologia. Fecha de consulta: 3 de mayo del 2018.

Snyder R, y Melo-Abreu JP (2010) Protección contra las heladas: fundamentos práctica y economía. Volumen. Organización de las Naciones Unidas para la Agricultura y la Alimentación. Roma, Italia. 242p

Thornthwaite CW (1948) An approach toward a rational classification of climate. Geographical Review 38: 55-94.

UNFPA (2018) Total population in millions, 2018. Fondo de Población de la Naciones Unidas. https://www.unfpa. org/es/data/world-population-dashboard. Fecha de consulta: 15 de mayo del 2018.

Vrieling A, de Leeuw J, Said M (2013) Length of growing period over Africa: Variability and Trends from 30 Years of NDVI time series. Remote Sensing 5: 982-1000. 